\title{
Identification of complete linkage disequilibrium in the DSG4 gene and its association with wool length and crimp in Chinese indigenous sheep
}

\author{
Y.H. Ling ${ }^{1,2 *}$, H. Xiang ${ }^{1,2 *}$, G. Zhang ${ }^{3}$, J.P. Ding ${ }^{1,2}$, Z.J. Zhang ${ }^{1,2}$, \\ Y.H. Zhang ${ }^{1,2}$, J.L. Han ${ }^{3}$, Y.H. Ma $^{3}$ and X.R. Zhang ${ }^{1,2}$ \\ ${ }^{1}$ College of Animal Science and Technology, Anhui Agricultural University, \\ Hefei, China \\ ${ }^{2}$ Local Animal Genetic Resources Conservation and Biobreeding Laboratory \\ of Anhui Province, Hefei, China \\ ${ }^{3}$ Institute of Animal Science, Chinese Academy of Agricultural Sciences, \\ Beijing, China \\ *These authors contributed equally to this study. \\ Corresponding author: J.P. Ding \\ E-mail: caaslyh@163.com
}

Genet. Mol. Res. 13 (3): 5617-5625 (2014)

Received October 7, 2013

Accepted January 13, 2014

Published July 25, 2014

DOI http://dx.doi.org/10.4238/2014.July.25.17

\begin{abstract}
The desmoglein 4 (DSG4) gene is a potential candidate in the search for genes that may affect wool traits, because of its function. This study aimed to screen for polymorphisms in partial exon 16 and 3'UTR of the sheep desmoglein 4 DSG4 gene, and to test its possible association with wool length and crimp associated with fur. Overall, 326 sheep were scanned via single-strand conformational polymorphism assay, through three pairs of primers. The breeds included Tan, Han, and TanxHan from China, Polled Dorset from Australia, and Suffolk from Britain genotypes $\mathrm{AA}, \mathrm{BB}$, and $\mathrm{AB}$ for primer2 and genotypes DD, EE, and DE for primer3 were detected in native breeds. Six SNPs
\end{abstract}


and 3-bp insertion/deletions were found in exon 16, of which 4 lead to amino acid substitutions. In addition, 1 SNP was found in 3'UTR. The DSG4 genotype was found to be strongly associated with all wool traits that were considered in this study $(\mathrm{P}<0.01)$. Sheep with the genotype MM had a higher least square mean compared to sheep with the genotype WW or WM with respect to birth scapular wool length ( $\mathrm{P}$ $<0.01)$, crimp number of birth scapular wool crimp $(\mathrm{P}<0.01)$, crimp number of weaning scapular wool crimp $(\mathrm{P}<0.01)$, and crimp number of weaning rump wool crimp $(\mathrm{P}<0.01, \mathrm{P}<0.05)$. In conclusion, our study is the first to demonstrate that the DSG4 gene may be a candidate, or major gene, which influences important wool traits.

Key words: Sheep; DSG4 gene; Polymorphisms; Wool length; Wool crimp; Complete linkage disequilibrium

\section{INTRODUCTION}

Through the candidate gene approach, many candidate or major genes have been identified in farm animals, some of which are being used in practical breeding programs by means of marker-assisted selection (MAS) or transgenosis. However, compared to other traits, markedly fewer candidate genes or molecular markers have been identified for wool traits in sheep (Purvis and Franklin, 2005; Zhang et al., 2011). Apart from genes coding wool components, genes that are highly expressed in the skin, which control the development and physiology of hair follicles, may be considered candidates influencing wool traits. Recent research has indicated that the desmoglein 4 (DSG4) gene may represent one such candidate gene.

Epidermal adhesion in the skin is mediated, in part, by specialized cell-cell junctions, named desmosomes (Chavez et al., 2012). These cell-cell junctions are characterized by the presence of desmosomal cadherins, termed as desmogleins and desmocollins (Simpson et al., 2011). In mice, desmoglein genes are composed of a group of 6 members, including DSG1-6, whereas DSG5 and DSG6 are not found in human or rat genomes (Zhang et al., 2008). A series of sequence variants of the $D S G 4$ gene have been reported to induce lanceolate hair in mice and rats (Meyer et al., 2004; Zhang et al., 2008). More recently, DSG4 gene mutations were found to be associated with human monilethrix hairs (Shimomura et al., 2006; Amagai, 2010). These studies convincingly demonstrate that DSG4 plays an important role in regulating the proliferation and differentiation of hair follicles in mammals (Schaffer et al., 2006; El-Amraoui and Petit, 2010). Although our knowledge about the sheep DSG4 gene remains limited, some DNA sequences of sheep that are homologous to this gene in other organisms are available in the GenBank. Furthermore, based on conserved synteny, this gene probably maps the region of microsatellite marker AGLA269 on OAR23 (Tetens et al., 2007; Zhang et al., 2011).

The Han sheep breed (small tailed) is used for its fur and meat, with this breed exhibiting high reproductive rates. Tan is the most important breed that is specialized for fur production, and has been designated as a nationally protected breed in China. The lamb fur of Tan is white colored; its wool staple twists tightly, and appears like a hair braid. Moreover, its fiber has a particular crimp with many bends. While the MAS has already made prominent genetic progress in animal breeding, the molecular markers associated with wool crimp have yet to be 
identified. This study was conducted for this purpose; hence, we primarily focused on the Tan breed in this experiment, investigating the association between DSG4 genotypes and crimp number in wool crimp.

\section{MATERIAL AND METHODS}

\section{Samples and traits}

A total of 326 individuals were used in the current study, including 118 Tan individuals from 7 sires, and 82 TanxHan individuals (67 F1 hybrids and 15 BC hybrids, 3/4 Tan + 1/4 Han) from Ningxia, 48 Han individuals from Shandong, and 48 Dorset individuals and 30 Suffolk individuals from Beijing. Measures of wool traits were conducted on Tan and TanxHan, including birth scapular wool length (BSL), crimp number of birth scapular wool crimp (BSC), crimp number of weaning scapular wool crimp (WSC), and crimp number of weaning rump wool crimp (WRC). BSL was based on the natural length; WSC and WRC were measured when the lambs were about 1 month old. The Dorset and Suffolk breeds were imported from Australia and Britain, respectively. The ear tissue of the investigated breeds was collected and stored at $-70^{\circ} \mathrm{C}$ in $75 \%$ ethanol before DNA extraction, as described by Sambrook and Russell (2001).

\section{Primer design}

A database search for the homologous sequences was performed by the BLASTN program (http://blast.ncbi.nlm.nih.gov/Blast.cgi), online. Two sheep ESTs from the skin (GenBank accession No. EE752650 and EE756890) were found respectively, which shared 96 and 95\% homolog with the reference RNA sequence (GenBank accession No. XM_617938) of the DSG4 gene (Bos taurus), corresponding to exon 16 and 3'UTR (Homo sapiens, GenBank accession No. AY177663). Three pairs of PCR primers were designed by Primer 5.0. based on the 2 sequences. Primer1 (forward: 5'-CCACGAAGGAGTAGGGTCT-3'; reverse: 5'-CCAAGTAAAGGGAGGTCAGT-3') was designed to amplify a 195-bp fragment covering the 94-288-bp region of EE752650. Primer2 (forward: 5'-GCACTGACCTCCCTTTACTT-3'; reverse: 5'-CAACCATCACTCACGCTACT-3') was designed to amplify a 364-bp fragment covering the 267-630-bp region of EE752650. Primer3 (forward: 5'-TAGTAGCGTGAGTGA TGGTT-3'; reverse: 5'-ATGTTGGTGATTACAAGGTG-3') was designed to amplify a 239bp fragment covering the 610-814 bp region of the EE752650 and 167-200-bp regions of EE756890. The total amplified region for the single-strand conformational polymorphism (SSCP) assay was $755 \mathrm{bp}$ in size, and began with 66 nucleotides of exon 16 and ended with 55 nucleotides of 3'UTR.

\section{Polymerase chain reaction (PCR)-SSCP}

PCR and SSCP were used to screen for polymorphisms within the amplified regions. The PCR was performed in a total volume of $15 \mu \mathrm{L}$, containing $100 \mathrm{ng}$ genomic DNA, 1X PCR buffer, $0.2 \mu \mathrm{M}$ of each primer, $200 \mu \mathrm{M}$ of each dNTP, and $0.75 \mathrm{U}$ Taq polymerase. SSCP conditions were as follows: $2 \mu \mathrm{L}$ PCR products were mixed with $8 \mu \mathrm{L}$ formamide loading dye for denaturation, samples were run on $12 \%$ non-denaturing acrylamide gel for 10-14 h at 
$4^{\circ} \mathrm{C}$ at $160-200 \mathrm{~V}$, and the gel was silver stained. PCR products with SSCP were purified and sequenced for the identification of polymorphisms.

\section{Statistical analysis}

Least square analysis was used to study the effects of genetic variants on wool length and crimp in Tan and TanxHan. The average number of each Tan sire was small, and the individuals studied were mostly ewes (only 4 rams were in the TanxHan breed group); thus, the effects of sire and sex were not considered in this study.

\section{RESULTS}

\section{Polymorphisms and frequency of genotype}

The SSCP-PCR showed that 4 genotypes were detected and defined as AA, BB, AB, and $\mathrm{BC}$ in primer2. Three genotypes were detected and defined as DD, EE, and DE in primer3. Table 1 shows the results of the genotype frequency distributions in the 5 study breeds. Interestingly, polymorphisms at the 2 loci were in complete linkage disequilibrium: AA was always linked to $\mathrm{DD}, \mathrm{BB}$ was always linked to $\mathrm{EE}$, and $\mathrm{AB}$ was also always linked to $\mathrm{DE}$, which were defined as composite genotypes WW, MM, and WM, respectively (genotype BC was not considered). The genotype $\mathrm{BC}$ was very scarce, and was only present in the Tan breed. The observed frequency of genotype MM (BB or EE) was small in Tan, Han, and TanxHan, while the genotypes MM and WM (AB or DE) were not observed in Dorset and Suffolk. Furthermore, 2 haplotypes were identified and defined as haplotypes $\mathrm{W}$ and $\mathrm{M}$; the latter haplotype had a relatively low frequency in native breeds, whereas it was not found in the Dorset and Suffolk breeds.

\begin{tabular}{|c|c|c|c|c|c|c|c|c|c|}
\hline \multirow[t]{2}{*}{ Population } & \multicolumn{4}{|c|}{ Genotype frequency of primer2 } & \multicolumn{3}{|c|}{ Genotype frequency of primer3 } & \multicolumn{2}{|c|}{ Haplotype frequency } \\
\hline & AA & BB & $\mathrm{AB}$ & $\mathrm{BC}$ & DD & $\mathrm{EE}$ & $\mathrm{DE}$ & W & $\mathrm{M}$ \\
\hline $\operatorname{Tan}(118)$ & $0.42(49)$ & $0.08(10)$ & $0.49(58)$ & $0.01(1)$ & $0.42(49)$ & $0.08(10)$ & $0.5(59)$ & 0.67 & 0.33 \\
\hline Han (48) & $0.65(31)$ & $0.04(2)$ & $0.31(15)$ & 0 & $0.65(31)$ & $0.04(2)$ & $0.31(15)$ & 0.80 & 0.20 \\
\hline TanxHan (82) & $0.52(43)$ & $0.1(8)$ & $0.38(31)$ & 0 & $0.52(43)$ & $0.1(8)$ & $0.38(31)$ & 0.71 & 0.29 \\
\hline Dorset (48) & 1 & 0 & 0 & 0 & 1 & 0 & 0 & 1 & 0 \\
\hline Suffolk (30) & 1 & 0 & 0 & 0 & 1 & 0 & 0 & 1 & 0 \\
\hline
\end{tabular}

Frequence of haplotype $\mathrm{W}$ and $\mathrm{M}$ also represents the frequence of allele $\mathrm{A}(\mathrm{D})$ and $\mathrm{B}(\mathrm{E})$, respectively (the frequence of allele $\mathrm{C}$ is not shown). The number of animals with every DSG4 genotype and sheep population are shown in parentheses.

Sequencing showed that haplotype W had 100\% homology with existing GenBank sequences (GenBank accession No. EE752650 and EE756890). Compared with haplotype W, in haplotype M, 7 polymorphisms were found, including 5 SNPs and a TTG insertion in exon 16 and 1 SNP in 3'UTR of the DSG4 gene, which were located at nucleotide positions 341(T/A), 353(G/A), 380(A/G), 601(C/T), 676(C/T), 723(TTG insertion), and 802(T/C), according to EE752650 (Figure 1A and B). Genotype BC was heterozygous at positions 341, 
353, 380, and 601. In addition, TC heterozygosis was present at position 390 (not shown). The TTG insertion induced predicted amino acid changes, namely, Ser/Ile substitution and Gly insertion. The SNPs at positions 341, 353, 380, and 390 induced predicted amino acid substitutions, namely, Phe/Ile, Val/Met, Arg/Gly, and Val/Ala, respectively. In comparison, 601(C/T) and $676(\mathrm{C} / \mathrm{T})$ were silent, based on the alignment of the CDS of the DSG4 gene of B. taurus, H. sapiens, Mus musculus, and Rattus norvegicus (GenBank accession No. XM_617938, AY227350, AY227349, and AY314982). The amplifier of primer1 showed no polymorphisms, and sequencing indicated that this primer had 100\% homology with EE752650.

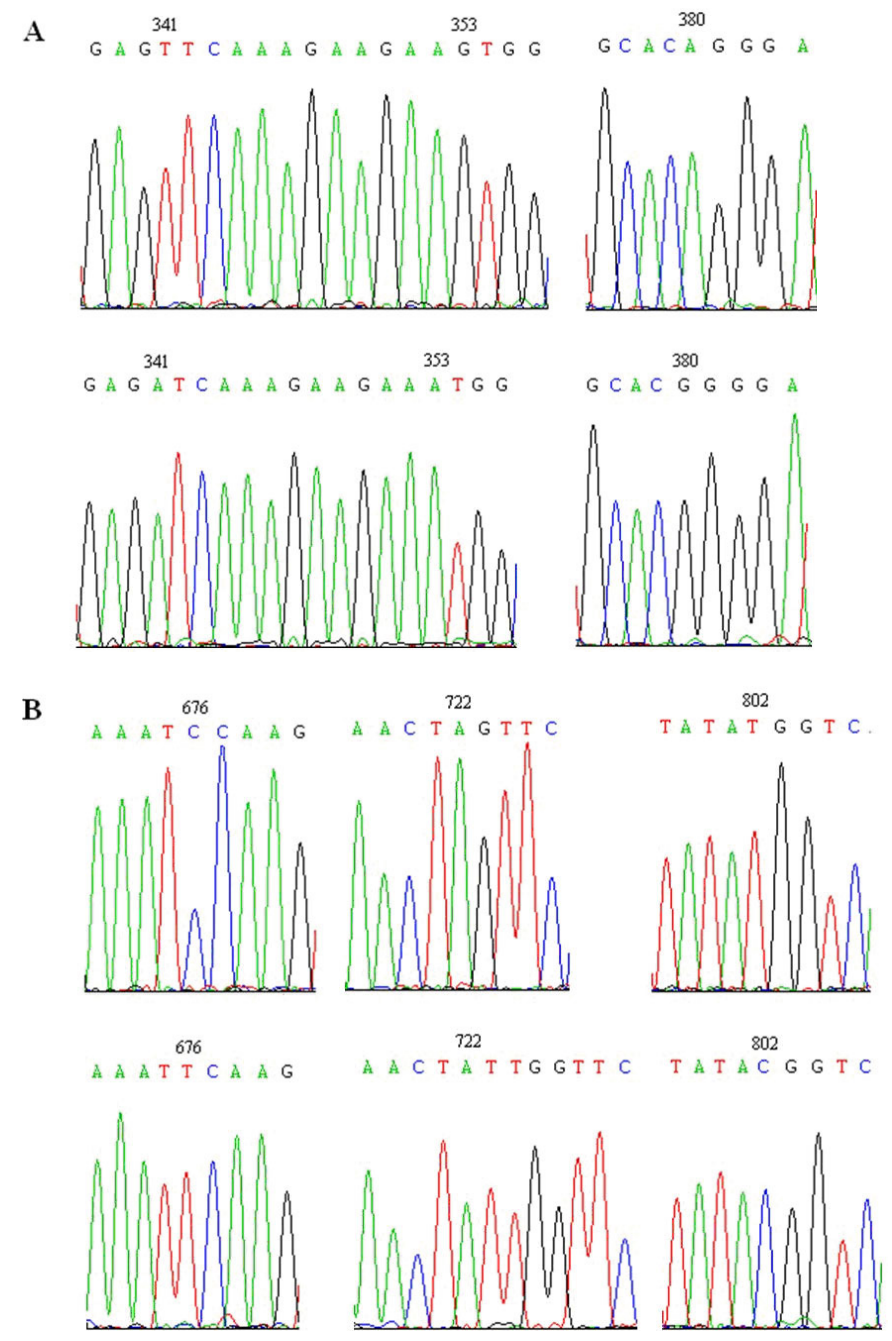

Figure 1. Schematic presentation of polymorphisms in the sheep $D S G 4$ gene. A. Sequence amplified by primer2 contains four polymorphisms, where AA genotype contains 341T353G380A601C (allele A), BB genotype contains 341A353A380G601T (allele B). B. Sequence amplified by primer3 contains three polymorphisms, where DD genotype contains 676C723TTGdeletion802T (allele D), EE genotype contains 676T723TTGinsertion802C (allele E). The sequence polymorphisms of $\mathrm{C}$ allele is not shown. 


\section{Association of the genotypes with wool length and crimp}

The GLM analysis showed that the DSG4 genotype has a highly significant effect on wool length and the crimp number of wool crimp (not shown, $\mathrm{P}<0.01)$. BSL $(\mathrm{P}<0.01$ ), BSC $(\mathrm{P}=0.001, \mathrm{P}<0.01)$, WSC $(\mathrm{P}<0.001, \mathrm{P}<0.01)$, and WRC $(\mathrm{P}<0.01, \mathrm{P}<0.05)$ were significantly higher in the MM genotype compared to the WW and WM genotypes, respectively (Table 2). However, no significant difference was observed between the WW and WM genotypes for BSL $(\mathrm{P}=0.91)$, BSC $(\mathrm{P}=0.38)$, WSC $(\mathrm{P}=0.19)$, and WRC $(\mathrm{P}=0.10)$, in which, the heterozygous genotype WM also appeared more advantageous for BSC, WSC and WRC compared to the WW genotype; however, the WM genotype showed no advantage for BSL. In addition, no significant interactions between genotype and breed were observed in the considered traits. The effect of the $\mathrm{BC}$ genotype was not analyzed because only 1 animal with this genotype was available. There were higher BSL $(\mathrm{P}<0.001)$, BSC $(\mathrm{P}>0.05)$, WSC $(\mathrm{P}<$ $0.001)$, and WRC $(\mathrm{P}<0.001)$ in Tan individuals compared to TanxHan individuals.

\begin{tabular}{|c|c|c|c|c|c|}
\hline \multirow[t]{2}{*}{ Trait } & \multicolumn{3}{|c|}{ Genotype } & \multicolumn{2}{|c|}{ Population } \\
\hline & WW & WM & MM & TanxHan & Tan \\
\hline BSL & $4.51 \pm 0.09^{\mathrm{A}}(87)$ & $4.49 \pm 0.10^{\mathrm{A}}(78)$ & $5.16 \pm 0.19^{\mathrm{B}}(18)$ & $4.46 \pm 0.12^{\mathrm{A}}(78)$ & $4.98 \pm 0.10^{\mathrm{B}}(105)$ \\
\hline BSC & $3.51 \pm 0.10^{\mathrm{A}}(87)$ & $3.64 \pm 0.11^{\mathrm{A}}(78)$ & $4.35 \pm 0.23^{\mathrm{B}}(18)$ & $3.69 \pm 0.14(78)$ & $3.97 \pm 0.12(105)$ \\
\hline WSC & $3.90 \pm 0.11^{\mathrm{A}}(86)$ & $4.12 \pm 0.12^{\mathrm{A}}(86)$ & $5.06 \pm 0.26^{\mathrm{B}}(16)$ & $3.92 \pm 0.15^{\mathrm{A}}(78)$ & $4.80 \pm 0.13^{\mathrm{B}}(110)$ \\
\hline WRC & $3.51 \pm 0.12^{\mathrm{Aa}}(74)$ & $3.79 \pm 0.12^{\mathrm{a}}(83)$ & $4.48 \pm 0.30^{\mathrm{Bb}}(14)$ & $3.48 \pm 0.19^{\mathrm{A}}(55)$ & $4.37 \pm 0.12^{\mathrm{B}}(116)$ \\
\hline
\end{tabular}

$\mathrm{BSL}=$ birth scapular wool length $(\mathrm{cm})$; BSC $=$ bends of birth scapular wool crimp; WSC = bends of weaning scapular wool crimp; WRC = bends of weaning rump wool crimp. Least square mean with different capital (lower case) superscripts within a trait differ significantly at $\mathrm{P}<0.01(\mathrm{P}<0.05)$, between the genotype or population classes. The number of animals used for GLM analysis are shown in parentheses (some records are unavailable).

\section{DISCUSSION}

\section{DSG4 gene, a novel candidate for wool traits}

The study of candidate genes is one of the primary methods used to locate specific genes related to certain wool traits in sheep (Purvis and Franklin, 2005). DSG4 plays an important role in regulating the development and differentiation of hair follicles (Owens et al., 2008; Zhang et al., 2008; Bazzi et al., 2009). Since the discovery of this gene (Kljuic et al., 2003), a number of mutations in the DSG4 gene have been reported to induce a group of hair disorders in mammals (Zhang et al., 2008; Amagai, 2010; El-Amraoui and Petit, 2010); thus, this gene may be considered as a potential candidate that influences wool traits. The human DSG4 is composed of 5 homologous extracellular domains, a transmembrane domain, and a carboxy-terminal cytoplasmic tail (Whittock and Bower, 2003). The human DSG4 gene is about $37 \mathrm{~kb}$ in size, and contains 16 exons and 15 introns. In addition, the cDNA $(3.6 \mathrm{~kb})$ contains an open reading frame of $3120 \mathrm{bp}$, which is especially expressed in the suprabasal epidermis and hair follicle (Owens et al., 2008; Bazzi et al., 2009; Amagai, 2010). Kljuic et al. (2003) identified an intragenic deletion encompassing exon 5-8 in the DSG4 gene, which un- 
derlies localized autosomal recessive hypotrichosis in humans, and has also been reported by several other studies (John et al., 2006; Amagai, 2010). Here, we identified a set of missense variants, including one 3-bp insertion in the coding sequence and 1 SNP in 3'UTR. These amino acid changes, which were located at the cytoplasmic tail, may change the function of DSG4 in some way. In addition, the SNP in 3'UTR might also influence the expression of this gene; however, these hypotheses require verification.

The two haplotypes (W and $\mathrm{M}$ ) identified here represent the first informative polymorphisms reported in the sheep $D S G 4$ gene. In addition, we provide primary evidence that the DSG4 genotype had a significant effect $(F$ test) on BSL $(\mathrm{P}<0.01)$, BSC $(\mathrm{P}<0.01)$, WSC $(\mathrm{P}<0.001)$, and WRC $(\mathrm{P}<0.01)$. Sheep with the MM genotype, which were the progeny of 6 Tan sires, exhibited significantly higher least squares mean in the considered traits $(\mathrm{P}<0.01$, $\mathrm{P}<0.05)$. Otherwise, it should be noted that the advantageous haplotype $(\mathrm{M})$ tends to act in a recessive manner, especially in BSL, which is worth further study in larger group.

Tan is a very special breed that is bred exclusively for its wool crimp; therefore, significantly higher WSC and WRC $(\mathrm{P}<0.001)$ were observed in Tan compared to TanxHan, whereas no significantly higher BSC was observed $(\mathrm{P}>0.05)$. This phenomenon may be explained by the fact that crimp number of wool crimp is a complicated and particular trait in Tan. Some genes controlling this characteristic may be expressed at low levels before birth, with expression gradually increasing until weaning. Alternatively, measurement error might be partly responsible for the obtained result.

This study aimed to identify useful molecular marker for MAS in the Tan breed, with the most important breeding aim being to increase crimp number of wool crimp in lamb fur. Although the association results showed that DSG4 gene might be a major gene that influences crimp number of wool crimp, the effect of using the $D S G 4$ marker (genotype $\mathrm{MM}$ ) in practical breeding by MAS must be tested in later generations, together with its low frequency. Hence, further studies are needed to confirm these preliminary results using larger breeds, or by multiplying the expression of this gene in the resource breed. We may further test the effect of the MM genotype on wool traits related to the lamb fur of the Han breed. If the results are confirmed, the low frequency of the MM genotype in the Tan and Han breeds indicates that it might represent a viable tool for MAS to improve the quality of lamb fur. In comparison, it is of interest to establish whether the MM genotype or M haplotype exist in Merino or other sheep breeds around the world, which are used for their wool or fur. Such information could be used to investigate the association of this genotype with wool length, yield, fiber diameter, and crimp. Therefore, our study provides important insights for potentially exploiting new markers for the molecular breeding of wool traits; however, to improve our understanding about the effect of the DSG4 gene on wool traits, research involving the cloning of the sheep $D S G 4$ gene, and screening for polymorphisms within the complete sequence, across a wider group of breeds is required.

\section{Origin hypotheses of the 2 haplotypes in the DSG4 gene}

Interestingly, we found complete linkage disequilibrium at the 2 loci studied in the $D S G 4$ gene in 5 sheep breeds ( 3 native and 2 western breeds; total $\mathrm{N}=326$ individuals). We found 2 haplotypes $(\mathrm{W}, \mathrm{M})$ in the Tan and Han breeds and their hybrids, whereas the M haplotype was not detected in the Dorset and Suffolk breeds; hence, 2 hypotheses may be proposed. 
The first hypothesis is that complete linkage disequilibrium may derive from 2 ancestral haplotypes (founder effect and genetic drift), because a number of alternative polymorphisms that are so tightly interlinked that they are unlikely to have arisen by sporadic crossing-over. This phenomenon may be directly supported by the fact that we identified haplotypes $\mathrm{W}$ and M (haplotype W) in 3 native breeds, including 326 animals, with just 1 other allele (C) being identified in 1 animal, which also showed no evidence of recombination.

There are 3 putative maternal lineages (also described by mitochondrial haplotypes A, $\mathrm{B}$, and $\mathrm{C}$ ) in sheep. Our previous study indicated that the frequencies of mitochondrial haplotypes $\mathrm{A}, \mathrm{B}$, and $\mathrm{C}$ were $55.3,28.0$, and $15.8 \%$ in $\operatorname{Han}(\mathrm{N}=38)$ and 30,70 , and $0 \%$ in Polled Dorset $(\mathrm{N}=30)$, respectively (Guo et al., 2005). In the present study, the observed frequency of DSG4 haplotype $\mathrm{W}$ and $\mathrm{M}$ was 80 and $20 \%$ in Han $(\mathrm{N}=48)$ and 100 and $0 \%$ in Polled Dorset $(\mathrm{N}=48)$, respectively. Therefore, this second hypothesis implies that maternal ancestors A and $\mathrm{B}$ carry the DSG4 haplotype $\mathrm{W}$, whereas the maternal ancestor $\mathrm{C}$ carries the DSG4 haplotype M. The maternal lineage $\mathrm{C}$ has been identified in sheep from China, Turkey, Caucasus, and Portugal (Pedrosa et al., 2005; Pereira et al., 2006; Tapio et al., 2006). Therefore, it would be of interest to investigate the linkage disequlibrium of the various sheep breeds of the world to establish whether the 2 haplotypes exist in Urial (Ovis vignei) and mouflon (Ovis musimon or Ovis orientalis), which might provide additional insights towards tracing the origins of modern sheep breeds, and the identification of wild ancestors.

\section{ACKNOWLEDGMENTS}

Research supported by the National Natural Science Foundation of China (\#31301934, \#31372310), the Natural Science Foundation of Anhui Province (\#1308085QC54), and the China Agriculture Research System (\#11004986) funds.

\section{REFERENCES}

Amagai M (2010). Autoimmune and infectious skin diseases that target desmogleins. Proc. Jpn. Acad. Ser. B Phys. Biol. Sci. 86: 524-537.

Bazzi H, Demehri S, Potter CS, Barber AG, et al. (2009). Desmoglein 4 is regulated by transcription factors implicated in hair shaft differentiation. Differentiation 78: 292-300.

Chavez MG, Buhr CA, Petrie WK, Wandinger-Ness A, et al. (2012). Differential downregulation of e-cadherin and desmoglein by epidermal growth factor. Dermatol. Res. Pract. 2012: 309587.

El-Amraoui A and Petit C (2010). Cadherins as targets for genetic diseases. Cold Spring Harb. Perspect. Biol. 2: a003095.

Guo J, Du LX, Ma YH, Guan WJ, et al. (2005). A novel maternal lineage revealed in sheep (Ovis aries). Anim. Genet. 36: 331-336.

John P, Tariq M, Arshad RM, Amin-Ud-Din M, et al. (2006). Recurrent intragenic deletion mutation in desmoglein 4 gene underlies autosomal recessive hypotrichosis in two Pakistani families of Balochi and Sindhi origins. Arch. Dermatol. Res. 298: 135-137.

Kljuic A, Bazzi H, Sundberg JP, Martinez-Mir A, et al. (2003). Desmoglein 4 in hair follicle differentiation and epidermal adhesion: evidence from inherited hypotrichosis and acquired pemphigus vulgaris. Cell 113: 249-260.

Meyer B, Bazzi H, Zidek V, Musilova A, et al. (2004). A spontaneous mutation in the desmoglein 4 gene underlies hypotrichosis in a new lanceolate hair rat model. Differentiation 72: 541-547.

Owens P, Bazzi H, Engelking E, Han G, et al. (2008). Smad4-dependent desmoglein-4 expression contributes to hair follicle integrity. Dev. Biol. 322: 156-166.

Pedrosa S, Uzun M, Arranz JJ, Gutierrez-Gil B, et al. (2005). Evidence of three maternal lineages in Near Eastern sheep supporting multiple domestication events. Proc. Biol. Sci. 272: 2211-2217.

Pereira F, Davis SJ, Pereira L, McEvoy B, et al. (2006). Genetic signatures of a Mediterranean influence in Iberian 
Peninsula sheep husbandry. Mol. Biol. Evol. 23: 1420-1426.

Purvis IW and Franklin IR (2005). Major genes and QTL influencing wool production and quality: a review. Genet. Sel. Evol. 37 (Suppl 1): S97-107.

Sambrook J and Russell D (2001). Molecular Cloning: A Laboratory Manual. 3rd edn. Cold Spring Harbor Laboratory Press, Cold Spring Harbor, New York.

Schaffer JV, Bazzi H, Vitebsky A, Witkiewicz A, et al. (2006). Mutations in the desmoglein 4 gene underlie localized autosomal recessive hypotrichosis with monilethrix hairs and congenital scalp erosions. J. Invest. Dermatol. 126: 1286-1291.

Shimomura Y, Sakamoto F, Kariya N, Matsunaga K, et al. (2006). Mutations in the desmoglein 4 gene are associated with monilethrix-like congenital hypotrichosis. J. Invest. Dermatol. 126: 1281-1285.

Simpson CL, Patel DM and Green KJ (2011). Deconstructing the skin: cytoarchitectural determinants of epidermal morphogenesis. Nat. Rev. Mol. Cell Biol. 12: 565-580.

Tapio M, Marzanov N, Ozerov M, Cinkulov M, et al. (2006). Sheep mitochondrial DNA variation in European, Caucasian, and Central Asian areas. Mol. Biol. Evol. 23: 1776-1783.

Tetens J, Goldammer T, Maddox JF, Cockett NE, et al. (2007). A radiation hybrid map of sheep chromosome 23 based on ovine BAC-end sequences. Anim. Genet. 38: 132-140.

Whittock NV and Bower C (2003). Genetic evidence for a novel human desmosomal cadherin, desmoglein 4. J. Invest. Dermatol. 120: 523-530.

Zhang G, Ma YH, Zhang YY and Guan WJ (2011). Six SNPs and a TTG indel in sheep desmoglein 4 gene are in complete linkage disequilibrium. Afr. J. Biotechnol. 10: 6852-6856.

Zhang MC, Furukawa H, Tokunaka K, Saiga K, et al. (2008). Mast cell hyperplasia in the skin of Dsg4-deficient hypotrichosis mice, which are long-living mutants of lupus-prone mice. Immunogenetics 60: 599-607. 